\title{
Application of Mobile Phases Containing Ionic Liquid for the Analysis of Selected Psychotropic Drugs by HPLC-DAD and HPLC-MS
}

\author{
A. Petruczynik*, K. Wróblewski and M. Waksmundzka-Hajnos \\ Department of Inorganic Chemistry, Medical University of Lublin, Chodźki 4a, 20-093 Lublin, Poland
}

Received: 16 May 2018; accepted: 16 May 2018

\begin{abstract}
In reversed-phase liquid chromatography, in case of the absence of additives, cationic basic compounds give rise to asymmetrical and broad peaks as a result of interactions of analyte cations with residual free silanols on silica-based stationary phases. Ionic liquids, added to the mobile phase, have been suggested as alternatives to amines to block the activity of free silanols. The different parameters affecting the retention behavior, symmetry of peak, system efficiency, and separation selectivity of selected psychotropic drugs, especially the effect of concentration of ionic liquid, kind and concentration of organic modifiers of mobile phases, and kind of stationary phases were investigated. The most selective and efficient systems are used for separations of psychotropic drug standards' mixture and for determination of selected psychotropic drugs in human serum.
\end{abstract}

Keywords: HPLC-DAD, psychotropic drugs, ionic liquid, serum sample

\section{Introduction}

Therapeutic drug monitoring of antidepressants provides the possibility to reduce side effects and optimize the treatment of patients with depression both to optimize therapy and to avoid medical complications, intoxication, non-response or non-compliance [1]. For this reason, it is advantageous to use a reliable, highly sensitive, selective, accurate and precise methods for determination of psychotropic drugs in biological samples.

Various chromatographic systems were applied for determination of psychotropic drugs in different biological samples. Most analysis of psychotropic drugs were performed on C18 stationary phases with various composition of mobile phases. Addition of acids or buffers at acidic $\mathrm{pH}$ to mobile phases containing organic modifier and water was often applied for determination of the drugs [2-4].

Mobile phases at basic $\mathrm{pH}$ were rarely used for analysis of these compounds [5]. Some authors reported application of mobile phases containing organic modifier, water, and salts, e.g., ammonium acetate [6]. Sometimes addition of amines as silanol blocker was applied [7].

Currently, silica as support matrix for bonded stationary phases in reversed-phase liquid chromatography is predominantly applied due to its high efficiency, good reproducibility, and universality [8]. Despite the many advantages, stationary phases bonded to the silica matrix have always free silanol groups, owing to steric problems in the derivatization process of silica supports. These silanols are negatively charged within the $\mathrm{pH}$ range commonly used in reversed-phase liquid chromatography (2.5-7.5) and can additionally interact with cationic compounds by ion-exchange interaction. Ion-exchange process is slow compared to the hydrophobic interaction of compounds with the groups bonded on the surface of the stationary phase. For this reason, the chromatographic peaks of basic compounds are broad and tailing and low system efficiency and poor separation selectivity are usually obtained. The suppression of the disadvantageous "silanol effect" is very important to obtain a good systems efficiency, separation selectivity of analytes, and appropriate peak shapes for basic compounds. Undesirable interactions with the silanols can be reduced by use of mobile phases containing buffers at low $\mathrm{pH}$, when sila-

*Author for correspondence: anna.petruczynik@umlub.pl nol ionisation is suppressed, or at high $\mathrm{pH}$, to suppress solute ionisation, as well as by addition of anionic ion-pair reagents to form neutral associates. Good peak symmetry and system efficiency for analysis of basic compounds are also achieved by use of mobile phases containing organic amines as silanol blockers.

To suppress free silanol groups, an addition of ionic liquids (ILs) to mobile phases can be also successfully applied $[9,10]$. The interaction mechanism in chromatographic systems with addition of ILs is more complex in comparison to the interactions in systems containing commonly used buffers or amines. Both cations and anions of ionic liquid play an important role in the retention of basic compounds. The cations of ionic liquids coated the surface of the stationary phase suppressing interactions with free silanol groups. Anions of ILs are also adsorbed on the surface of stationary phase and thus, with ionic liquid (IL) cations creating a bilayer, positively or negatively charged, improved the shape of peaks of analytes.

Many biological active compounds, e.g., components of plant extracts, and various drugs contain basic groups in their structure and in reversed-phase liquid chromatography (RPLC) are analyzed with application of mobile phases containing addition of ionic liquids as silanol blocker. The use of ILs additives was reported for analysis of some alkaloids, e.g., fangchinoline and tetrandrine in Stephania tetrandra [9], jatrorrhizine, palmatine, and berberine in Huangbo, the dried bark of Chinese Corktree [10]. ILs were also applied for analysis of some basic drugs such as $\beta$-blockers [11] and $\beta$-lactam antibiotics [12].

Besides the commonly used $n$-alkyl-type reversed-phase columns containing $n$-alkyl-type ligands onto a silica support, alternative hybrid RP-type phases providing additional interaction sites and properties due to embedded functional groups can be used $[13,14]$. The introduction of hydrophobic $\pi-\pi$ active aromatic moieties to the common $n$-alkyl chain RP-sites generates a concerted $\pi-\pi$ reversed-phase retention mechanism, which is a consequence of the new functionality. These interactions are the type of electron donor-acceptor interaction between $\pi$ electrons of the phenyl groups bonded on the stationary phase and the $\pi$ electrons of the aromatic moieties of analyzed compounds. The $\pi-\pi$ interactions may limit the ion exchange process on free silanol groups because of the preferred interactions of basic analytes with the phenyl. The $\pi-\pi$ interactions typically involve the charge transfer of electrons from electron-rich ( $\pi$-base) to

This is an open-access article distributed under the terms of the Creative Commons Attribution-NonCommercial 4.0 International License (https://creativecommons.org/licenses/by-nc/4.0/), which permits unrestricted use, distribution, and reproduction in any medium for non-commercial purposes, provided the original author and source are credited, a link to the CC License is provided, and changes - if any - are indicated. 
electron-poor ( $\pi$-acid) substances. The $\pi-\pi$ interactions can also involve a simple overlap of $\pi$-orbitals in two interacting molecules. Ether linkage to alkyl chain (as in Polar RP column) increases aromaticity of the phenyl group and also provides $\pi-\pi$ interactions between the phenyl ligands of stationary phase and aromatic moieties of solutes.

The aim of the paper is investigation of influence of IL applied as mobile phases additive on phenyl stationary phase (RP-Phe column) for twenty-two basic psychotropic drugs retention behavior. The effect of the concentration of IL or acetonitrile in mobile phases and effect of temperature was investigated and optimal eluent for analysis of psychotropic drugs in human serum was chosen.

\section{Experimental}

Chemicals and Drug Standards. Acetonitrile, methanol, tetrahydrofuran, dioxane, and 1-butyl-3-methylimidazolium tetrafluoroborate of chromatographic quality were from E. Merck (Darmstadt, Germany). Formic acid was of p.a. quality (Polish Reagents Gliwice, Poland).

Drug standards were obtained from the following sources: aripiprazole, Otsuka Pharmaceutical Europe Ltd. (Wexham, Great Britain); chlordiazepoxide, Tarachomińskie Zakłady Farmaceutyczne "Polfa" S. A. (Warsaw, Poland); citalopram, Ranbaxy Sp. z o. o. (Warsaw, Poland); desipramine hydrochloride, Temmler Pharma GmbH \& Co. KG (Marburg, Germany); escitalopram and paroxetine, Gedeon Richter (Grodzisk Mazowiecki, Poland); carbamazepine, Sigma-Aldrich (Saint Louis, USA); clozapine, EGIS Pharmaceuticals PLC (Budapest, Hungary); quetiapine, Adamed Sp. z o.o. (Pieńków, Poland); levomepromazine, PROTERAPIA Sp. z o.o. (Warsaw, Poland); lamotrigine, Glenmark Pharmaceuticals (Prague, Czech Republic); moclobemide, Hoffmann LaRoche (Basel, Switzerland); mirtazapine, Orion Corporation (Espoo, Finland); olanzapine, Actavis Group PTC (Hafnarfjörður, Iceland); oxcarbazepine, Generics UK Ltd. (Potters Bar, Great Britain); perazine, HASCO-LEKS.A. (Wrocław, Poland); perphenazine, MSD (Warsaw, Poland); risperidone, Janssen-Cilag International NV (Beerse, Belgium); sertraline, Biofarm (Poznań, Poland); venlafaxine, Polfa Pabianice S.A. (Pabianice, Poland); opipramol dihydrochloride; and zolpidem tartas, Polpharma (Stargard Gdański, Poland).

Apparatus and HPLC Conditions. The high-performance liquid chromatography with diode array detection (HPLC-DAD) analysis was performed using liquid chromatograph LC-10 ATVP Shimadzu equipped with column Synergi Polar RP 80A (RP-Phe column) $150 \times 4.6 \mathrm{~mm}, 5 \mu \mathrm{m}$. Chromatograph was equipped with Shimadzu detector SPD 10 AVVP and Rheodyne $20 \mu \mathrm{L}$ injector. The detection was carried out at $240 \mathrm{~nm}$ wavelength. All chromatographic measurements were controlled by CTO-10ASVP thermostat. Flow rate was $1.0 \mathrm{~mL} / \mathrm{min}$.

Preparation of Serum Samples. Samples were collected from psychiatric patients. After blood coagulation, the samples were centrifuged for $10 \mathrm{~min}$ at $1500 \times \mathrm{g}$. The serum was separated and stored at $-20{ }^{\circ} \mathrm{C}$ until analysis.

Solid-phase extraction (SPE) for serum sample preparation was performed using BAKERBOND ${ }^{\mathrm{TM}}$ spe Octadecyl (C18) J.T. Baker (Phillipsburg, USA) cartridges $(100 \mathrm{mg} / 1 \mathrm{~mL})$ and SPE chamber-Baker SPE-12G, J.T. Baker (Philipsburg, USA).

Serum sample $(1.5 \mathrm{~mL})$ was diluted with $1.5 \mathrm{~mL}$ of ammonium buffer at $\mathrm{pH}$ 8.6. SPE column was activated with $1 \mathrm{~mL}$ of methanol and conditioned with $1 \mathrm{~mL}$ of mixture containing water and ammonium buffer at $\mathrm{pH} 8.6$ in ratio 5:1. Then, the serum sample was introduced to the column at speed $1 \mathrm{~mL} / \mathrm{min}$. The column was prewashed with $1 \mathrm{~mL}$ of methanol solution $(25 \% \mathrm{v} / \mathrm{v})$ and dried applying vacuum for $3 \mathrm{~min}$. The extracted drugs were eluted twice with $1 \mathrm{~mL}$ of mixture containing $98 \%$ methanol and $2 \%$ acetic acid. The eluted sample was evaporated to dryness and dissolved in $0.5 \mathrm{~mL}$ of methanol. Next, $20 \mu \mathrm{L}$ of the sample was injected into the HPLC column.

\section{Results and Discussion}

Psychotropic drug standards (Table 1) were chromatographed on RP-Phe column in eluent systems containing organic modifier, water, and ionic liquid 1-butyl-3-methylimidazolium tetrafluoroborate as free silanol blocker. Tested chromatographic systems were compared in terms of retention of investigated compounds, peak shape, system efficiency, and differences in separation selectivity. In the first step of investigations, commonly used mobile phase containing acetonitrile, water, and formic acid and mobile phase containing acetonitrile, water, and IL were applied. For comparison of chromatographic systems performance, important parameters, theoretical plate number and asymmetry factor, were calculated. Theoretical plate number $(N)$ is an index that indicates chromatographic system efficiency and is calculated by Eq. (1).

$$
N=5.54 x\left(\frac{t_{R}}{w_{0.5 h}}\right)
$$

Table 1. $t_{\mathrm{R}}$ and $N / m$ values obtained for psychotropic drugs on Polar RP column with mobile phase containing $25 \%$ MeCN, water, and different concentrations of IL

\begin{tabular}{|c|c|c|c|c|c|c|c|c|c|c|c|c|c|c|c|c|}
\hline \multirow{2}{*}{$\begin{array}{l}\text { Name of } \\
\text { compounds }\end{array}$} & \multicolumn{2}{|c|}{$0 \%$} & \multicolumn{2}{|c|}{$0.05 \%$} & \multicolumn{2}{|c|}{$0.1 \%$} & \multicolumn{2}{|c|}{$0.25 \%$} & \multicolumn{2}{|c|}{$0.5 \%$} & \multicolumn{2}{|c|}{$1.0 \%$} & \multicolumn{2}{|c|}{$1.5 \%$} & \multicolumn{2}{|c|}{$2.0 \%$} \\
\hline & $t_{\mathrm{R}}$ & $N / m$ & $t_{\mathrm{R}}$ & $N / m$ & $t_{\mathrm{R}}$ & $N / m$ & $t_{\mathrm{R}}$ & $N / m$ & $t_{\mathrm{R}}$ & $N / m$ & $t_{\mathrm{R}}$ & $N / m$ & $t_{\mathrm{R}}$ & $N / m$ & $t_{\mathrm{R}}$ & $N / m$ \\
\hline & & & 68 & & & & & & .924 & & 9.743 & & 9.743 & & 73.746 & \\
\hline Chlordiazepoxide & & & & & & & & & & & & & & & & 36,900 \\
\hline Citalopram & 14.872 & 15,300 & 9.033 & 3,100 & 9.442 & 7,300 & 21.565 & 30,400 & 20.678 & 33,100 & 21.394 & 32,200 & 21.394 & 30,700 & 19.568 & 29,800 \\
\hline imipramine & 0.158 & 9800 & 4.807 & 7,000 & 5.371 & 18,900 & 8.344 & 46,900 & 26.787 & 41,100 & 26.390 & 37,400 & 26.390 & 33,600 & 24.899 & 31,400 \\
\hline & .843 & 22,000 & .136 & 7,200 & 25 &, 000 & 10 & 600 & .689 & 00 & 80 & 00 & 80 & 25,300 & 600 & 21,600 \\
\hline & &, 800 & & & & & & & & & & & & 000 & & 8,300 \\
\hline & .928 & 19,300 & 4.082 & 40,900 & 6.315 & 26,000 & 15.191 & 37,000 & 12.199 & 32,900 & 3.425 & 29,800 & 13.425 & 26,900 & 286 & 25,700 \\
\hline ine & & 14,700 & 7.291 & 00 & 23 & 19,9 & 35 & 3,900 & & 49,000 & 73 & 38,100 & 573 & 30,500 & 16.295 & 25,800 \\
\hline romazine & & 100 & .722 & & & & & & & & & & & & & 600 \\
\hline & & 10,800 & 4.759 & 25,100 & & & 69 & 00 & & & & 100 & & & & 900 \\
\hline & & 9500 & 4.503 & 19,600 & & & & & & & & & & 17,600 & &, 100 \\
\hline & & 7200 & 6.895 & 14,700 & 7.5 & 25,1 & 7.048 & 27, & 6. & 17,7 & 10 & 00 & 6.910 & 20,500 & 76 & 23,100 \\
\hline & & 4800 & 3.492 & 400 & & & 3.374 & & & & 68 & 800 & 3.468 & 00 & & 300 \\
\hline & .321 & 15,900 & 21.218 & 32,500 & 21.594 & 35,000 & 21.266 & 50,200 & 06 & 500 & 503 & 40,300 & 19.503 &, 200 & 959 & 7,000 \\
\hline $\mathrm{Ox}$ & & 1,100 & .687 & 43,900 & 9.933 & 4,200 & 916 &, 300 & & 200 & 29 & 40,7 & 8.829 & 37,300 & 9.194 & 34,000 \\
\hline & & 8,500 & 1.251 & & 3.305 & & 35.739 & & 33.600 & & 2.985 & & 32.985 & 100 & 107 & 31,200 \\
\hline & 7.129 & 17,800 & 53.601 & 35,800 & 4.689 & 40,200 & 37.602 & 65,300 & 36.376 & 72,000 & 38.041 & 63,500 & 38.041 & 54,700 & 41.692 & 49,500 \\
\hline & & & & & & & & & & & & & & & & 38,200 \\
\hline & .158 & 10,100 & 46.871 & 25,400 & 49.045 & 24,200 & 55.726 & & 50.578 & & 47.987 & 55,200 & 47.987 & 51,400 & 49.008 & 48,500 \\
\hline faxine & 6.722 & 11,200 & 7.203 & 30,900 & 6.562 & 31,900 & 6.849 & 24,400 & 6.685 & 16,100 & 6.829 & 14,900 & 6.829 & 11,900 & 7.024 & 10,200 \\
\hline Zolpidem & 11.125 & 9800 & 13.554 & 21,900 & 10.665 & 18,900 & 9.770 & 41,100 & 9.624 & 35,900 & 9.322 & 32,200 & 9.322 & 29,100 & 9.044 & 27,200 \\
\hline
\end{tabular}


where $t_{\mathrm{R}}$ is retention time and $w_{0.5 \mathrm{~h}}$ is the peak width measured at 0.5 of peak high.

Asymmetry factor $\left(A_{\mathrm{s}}\right)$ is a coefficient that shows the degree of peak symmetry and is calculated by Eq. (2):

$$
A_{s}=\frac{b}{a}
$$

where $b$ is distance from the point at peak midpoint to the trailing edge (measured at $10 \%$ of peak height) and $a$ is distance from the leading edge of peak to the midpoint (measured at $10 \%$ of peak height).

In system containing only acetonitrile, water, and formic acid, for most investigated drug, peaks were very wide and asymmetric; only for six out of twenty-two drugs, $A_{\mathrm{s}}$ values were in acceptable range $\left(0.8<A_{\mathrm{s}}<1.5\right)$. System efficiency in the mobile phase was also poor for most investigated compounds, only for seven drugs $N / m$ values were higher than 25,000 . The significant improvement of peak shape and systems efficiency was obtained in mobile phase system containing acetonitrile, water, and

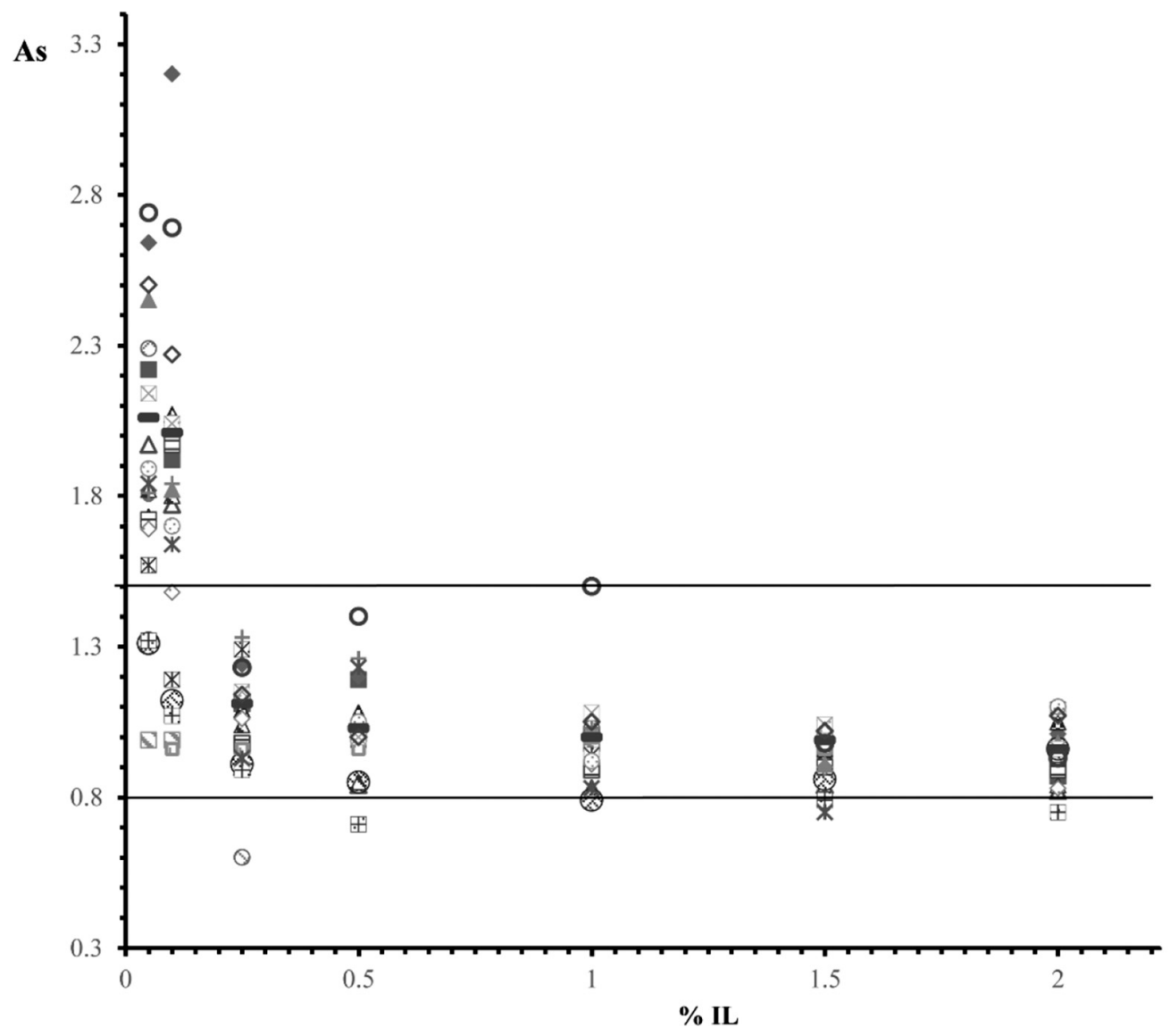

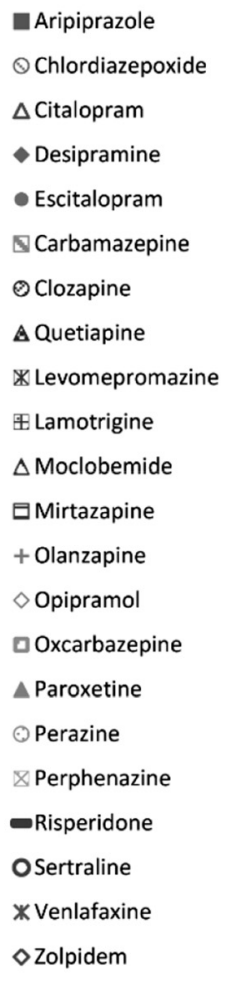

Figure 1. Graphical comparison of $A_{\mathrm{s}}$ values of psychotropic drugs on the RP-Phe column with eluent systems containing $25 \% \mathrm{MeCN}$, water, and various concentrations of 1-butyl-3-methylimidazolium tetrafluoroborate

Table 2. $A_{\mathrm{s}}$ and $N / m$ values obtained for psychotropic drugs on Polar RP column with mobile phase containing different concentration of MeCN, water, and $0.25 \%$ IL

\begin{tabular}{|c|c|c|c|c|c|c|c|c|c|c|c|c|c|c|c|c|}
\hline \multirow{2}{*}{$\begin{array}{l}\text { Name of } \\
\text { compounds }\end{array}$} & \multicolumn{2}{|c|}{10} & \multicolumn{2}{|c|}{15} & \multicolumn{2}{|c|}{20} & \multicolumn{2}{|c|}{25} & \multicolumn{2}{|c|}{30} & \multicolumn{2}{|c|}{35} & \multicolumn{2}{|c|}{40} & \multicolumn{2}{|c|}{45} \\
\hline & $A_{\mathrm{s}}$ & $N / m$ & $A_{\mathrm{s}}$ & $N / m$ & $A_{\mathrm{s}}$ & $N / m$ & $A_{\mathrm{S}}$ & $N / m$ & $A_{\mathrm{s}}$ & $\mathrm{N} / \mathrm{m}$ & $A_{\mathrm{s}}$ & $N / m$ & $A_{\mathrm{S}}$ & $N / m$ & $A_{\mathrm{S}}$ & $N / m$ \\
\hline Aripiprazole & - & & - & & - & & 1.11 & 64,300 & 1.12 & 58,500 & 1.17 & 56,300 & 1.19 & 47,900 & 1.23 & 43,700 \\
\hline Chlordiazepoxide & - & & 0.98 & 59,500 & 0.68 & 40,400 & 0.60 & 12,600 & 0.66 & 22,100 & 1.59 & 17,800 & 1.66 & 19,100 & - & \\
\hline Citalopram & - & & - & & 0.89 & 40,400 & 0.90 & 30,400 & 0.93 & 29,100 & 1.20 & 42,700 & 1.05 & 31,200 & - & \\
\hline Desimipramine & - & & - & & 1.24 & 52,400 & 1.24 & 46,900 & 1.10 & 42,300 & 1.24 & 45,800 & 1.35 & 38,800 & 1.37 & 34,500 \\
\hline Escitalopram & - & & - & & 0.93 & 43,400 & 0.92 & 36,600 & 0.91 & 27,400 & 1.07 & 35,800 & 1.06 & 29,200 & - & \\
\hline Carbamazepine & - & & - & & 0.95 & 57,900 & 0.97 & 54,500 & 1.00 & 50,100 & 1.03 & 47,100 & 1.09 & 47,500 & 1.14 & 40,800 \\
\hline Clozapine & - & & 0.92 & 45,000 & 0.97 & 48,900 & 0.91 & 37,000 & 0.93 & 35,400 & 1.08 & 67,000 & 1.19 & 18,700 & - & \\
\hline Quetiapine & - & & - & & 1.06 & 51,100 & 1.09 & 43,900 & 1.06 & 33,500 & 1.22 & 46,000 & 1.24 & 36,700 & - & \\
\hline Levomepromazine & - & & - & & - & & 1.29 & 56,800 & 1.22 & 50,900 & 1.30 & 51,400 & 1.50 & 51,400 & 1.40 & 39,700 \\
\hline Lomatrigine & 0.75 & 22,900 & 0.75 & 18,800 & 0.78 & 15,500 & 0.89 & 17,500 & 0.85 & 17,500 & 1.19 & 29,900 & - & & - & \\
\hline Maclobenid & 1.14 & 16,900 & 0.79 & 15,500 & 1.18 & 31,000 & 1.04 & 22,700 & 1.12 & 24,600 & 1.30 & 25,600 & - & & - & \\
\hline Mirtazapine & 0.97 & 36,500 & 1.28 & 40,000 & 1.11 & 15,800 & 0.98 & 27,100 & 1.03 & 28,900 & 1.61 & 27,200 & - & & - & \\
\hline Olanzapine & 0.62 & 4100 & 1.07 & 23,400 & 1.14 & 19,000 & 1.33 & 8700 & 1.35 & 20,400 & 1.34 & 14,200 & - & & - & \\
\hline Opipramol & - & & - & & 1.14 & 57,200 & 1.06 & 50,200 & 1.03 & 42,500 & 1.22 & 43,100 & 1.27 & 36,200 & - & \\
\hline Oxcarbamazepine & - & & 0.97 & 51,700 & 0.95 & 48,900 & 0.96 & 45,300 & 1.01 & 43,300 & 1.04 & 41,000 & - & & - & \\
\hline Paroxetine & - & & - & & - & & 1.14 & 48,600 & 1.13 & 43,500 & 1.18 & 52,000 & - & & - & \\
\hline Perfenazine & - & & - & & - & & 1.15 & 65,300 & 1.16 & 58,500 & 1.28 & 53,100 & 1.10 & 30,900 & 1.29 & 38,300 \\
\hline Risperidon & - & & 1.13 & 53,600 & 1.13 & 49,300 & 1.11 & 43,100 & 1.13 & 40,400 & 1.20 & 39,500 & 1.07 & 33,700 & - & \\
\hline Sertraline & - & & - & & - & & 1.23 & 57,800 & 0.98 & 57,700 & 1.45 & 55,200 & 1.38 & 31,600 & 1.40 & 22,600 \\
\hline Venlafaxine & 0.95 & 34,400 & 0.81 & 36,700 & 0.85 & 24,800 & 0.93 & 24,400 & 0.95 & 21,900 & 1.14 & 31,600 & - & & - & \\
\hline Zolpidem & & & 1.15 & 47,800 & 1.08 & 40,400 & 1.14 & 41,100 & 1.15 & 38,800 & 1.31 & 37,100 & 1.32 & 33,100 & - & \\
\hline
\end{tabular}

- , Very stong or weakly retention. 


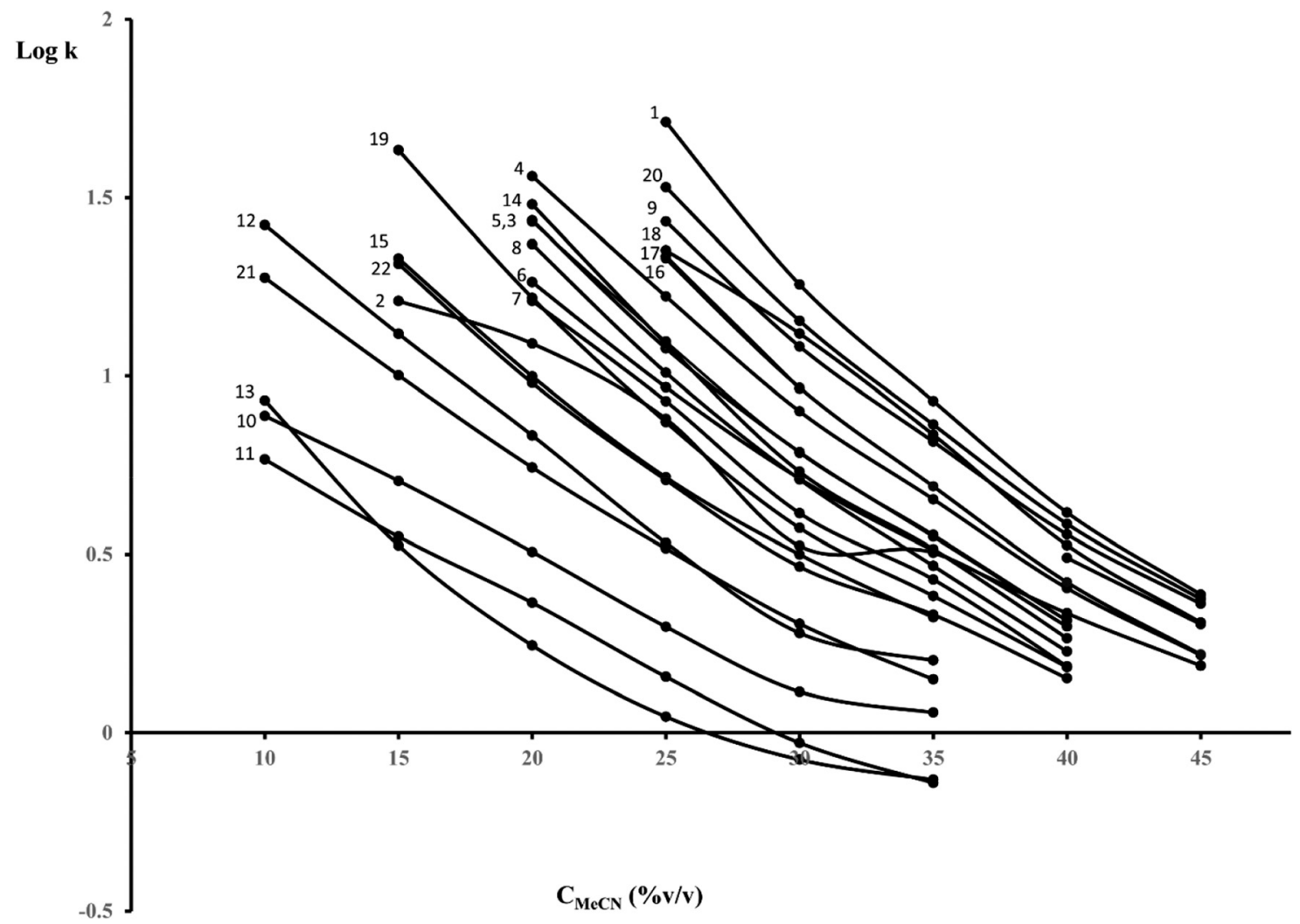

Figure 2. The dependencies of psychotropic drugs $\log k$ values versus concentration of acetonitrile. System: RP-Phe column eluted with a mixture of $\mathrm{MeCN}$, water, and $0.5 \%$ 1-butyl-3-methylimidazolium tetrafluoroborate

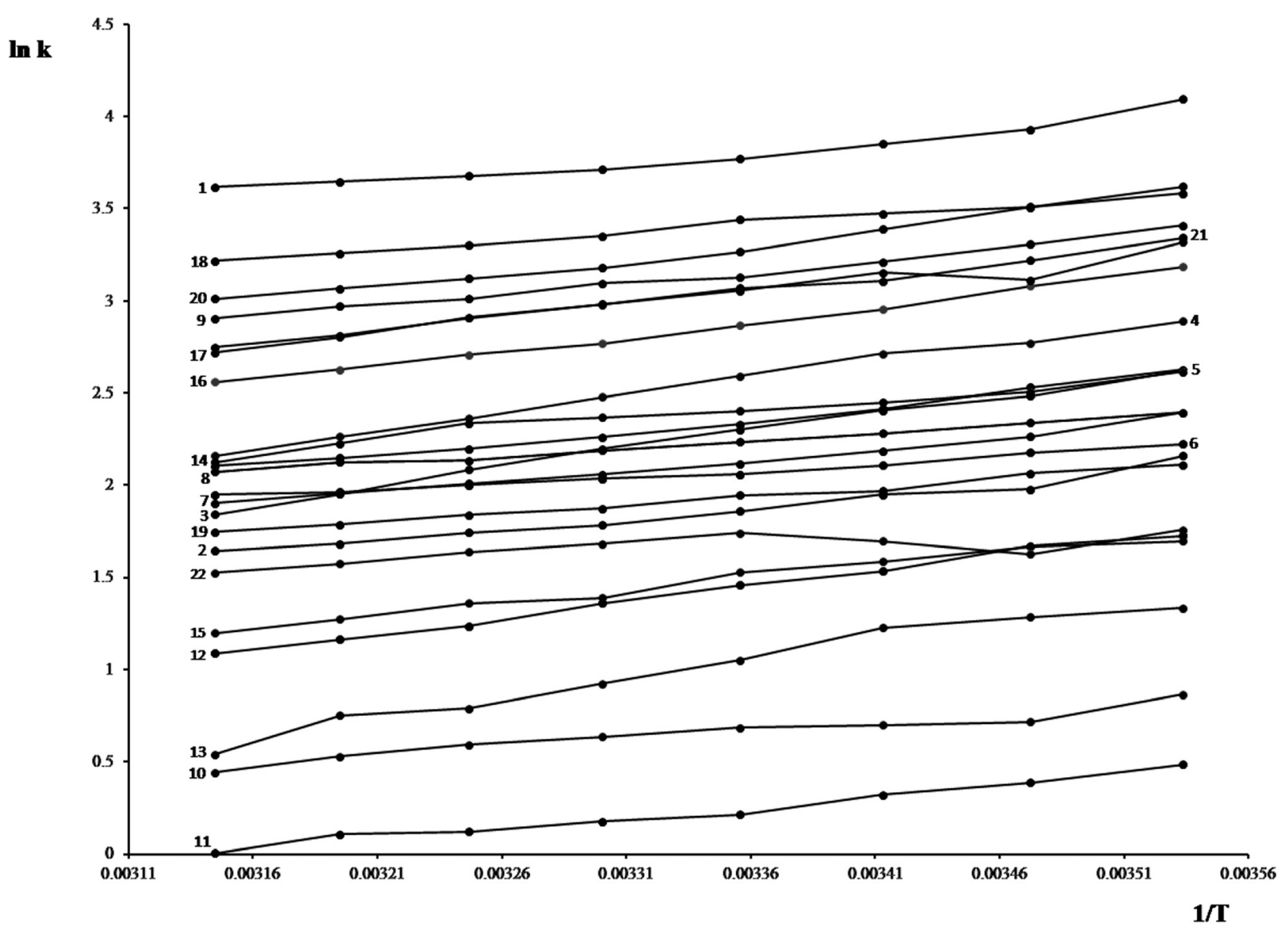

Figure 3. The dependencies of psychotropic drugs $\log k$ values versus temperature on the RP-Phe column eluted with a mixture of MeCN, water, and $0.5 \%$ 1-butyl-3-methylimidazolium tetrafluoroborate 
addition of even small amount of IL $(0.05 \%)$. In mobile phases containing IL, significantly higher theoretical plate number $(N)$ was also obtained. In eluent system containing only $0.05 \%$ IL for fifteen out of twenty-two investigated psychotropic drugs, $N / m$ was higher than 25,000 .

Further increase in IL concentration resulted in further changes in retention, peaks' symmetry, and system efficiency. The retention was slightly decreased with the increase of IL concentration for most investigated compounds. The separation selectivity for all drugs was similar in all range of IL concentration. However, great differences were obtained in peaks' symmetry with the change in IL concentration for investigated drugs (Figure 1). In systems with $0.25 \%$ or higher concentration of IL, peaks for almost all investigated drugs were symmetrical. For example, in system containing $0.25 \% \mathrm{IL}$, symmetrical peaks were obtained for 21 drugs, except chlordiazepoxide, and for 16 drugs symmetry of peaks was excellent; in system with $0.5 \% \mathrm{IL}$, only for lomatrigine, peak was asymmetrical, and for 14 compounds, symmetry was excellent. Slight improvement of peaks shape was obtained when mobile phases containing higher concentration of IL were applied. In system containing $1 \%$ of $\mathrm{IL}$, excellent peak symmetry was obtained for 15 compounds, in system with $1.5 \%$ for 17 and in system containing $2 \%$ of IL for 15 investigated drugs. In mobile phase systems containing 1-2\% IL for all investigated drugs, peaks of $A_{\mathrm{s}}$ values were in acceptable range.

Significant increase of systems' efficiency was observed in eluent containing only $0.05 \%$ IL in comparison to system without the additive (Table 1). An increase of the IL concentration caused increase of systems efficiency for all investigated psychotropic drugs. For most investigated drugs, the highest $N / m$ values were obtained for eluent systems containing $0.1-0.5 \% \mathrm{IL}$. On the basis of the obtained results, in further investigations, mobile phases containing $0.25 \%$ IL were applied.

In the next step of experiments, an effect of acetonitrile concentrations in aqueous mobile phases containing water and $0.25 \%$ IL on chromatographic parameters was examined (Figure 1, 2 and Table 2). Retention of psychotropic drugs decreased with increase of organic modifier concentrations in the investigated range of $10-45 \% \mathrm{MeCN}$. Separation selectivity also decreased with the increase of $\mathrm{MeCN}$ concentration for most investigated drugs. In systems with 10 or $15 \% \mathrm{MeCN}$, most drugs are strongly retained on RP-Phe column.

With the change of $\mathrm{MeCN}$ concentration, changes in peak symmetry and systems efficiency were also observed. However,

Table 3. $A_{\mathrm{s}}$ and $\mathrm{N} / \mathrm{m}$ values obtained for psychotropic drugs on Polar RP column with mobile phase containing $25 \% \mathrm{MeCN}$, water, and $0.25 \%$ IL at different temperature

\begin{tabular}{|c|c|c|c|c|c|c|c|c|c|c|c|c|c|c|c|c|}
\hline \multirow{2}{*}{$\begin{array}{l}\text { Name of } \\
\text { compounds }\end{array}$} & \multicolumn{2}{|c|}{$10^{\circ} \mathrm{C}$} & \multicolumn{2}{|c|}{$15^{\circ} \mathrm{C}$} & \multicolumn{2}{|c|}{$20^{\circ} \mathrm{C}$} & \multicolumn{2}{|c|}{$25^{\circ} \mathrm{C}$} & \multicolumn{2}{|c|}{$30{ }^{\circ} \mathrm{C}$} & \multicolumn{2}{|c|}{$35^{\circ} \mathrm{C}$} & \multicolumn{2}{|c|}{$40^{\circ} \mathrm{C}$} & \multicolumn{2}{|c|}{$45^{\circ} \mathrm{C}$} \\
\hline & $A_{\mathrm{s}}$ & $N / m$ & $A_{\mathrm{s}}$ & $N / m$ & $A_{\mathrm{s}}$ & $N / m$ & $A_{\mathrm{s}}$ & $N / m$ & $A_{\mathrm{s}}$ & $N / m$ & $A_{\mathrm{s}}$ & $N / m$ & $A_{\mathrm{s}}$ & $N / m$ & $A_{\mathrm{s}}$ & $N / m$ \\
\hline Aripiprazole & 1.20 & 55,300 & 1.16 & 58,400 & 1.01 & 61,000 & 1.12 & 64,800 & 1.21 & 61,200 & 1.35 & 59,700 & 1.39 & 56,200 & 1.51 & 54,200 \\
\hline Chlordiazepoxide & .73 & 10,400 & 1.54 & 0,700 & 1.35 & 2,400 & .90 & 12,600 & 0.78 & 12,700 & 0,76 & 12,100 & 0.74 & 11,300 & 0.73 & 10,300 \\
\hline Citalopram & 1.21 & 39,600 & 1.17 & 36,800 & 1.10 & 32,300 & 0.90 & 30,1 & 0.83 & 28,100 & 0.80 & 25,900 & 0.79 & 22,800 & 0.74 & 22,300 \\
\hline Desimipramine & 19 & 45,700 & 1.10 & 46,200 & 1.13 & 46,300 & 1.24 & 46,900 & 1.46 & 45,200 & 1.53 & 43,200 & 1.57 & 40,700 & 1.62 & 40,100 \\
\hline Escitalopram & 0.93 & 34,000 & 1.03 & 34,800 & 1.01 & 35,200 & 0.90 & 36,300 & 0.81 & 36,700 & 0.79 & 35,700 & 0.75 & 34,200 & 0.72 & 33,300 \\
\hline Carbamazepine & .03 & 41,900 & 1.06 & 45,800 & 1.02 & 48,000 & 0.97 & 53,900 & 0.90 & 52,100 & 0.85 & 50,800 & 0.81 & 45,600 & 0.79 & 41,800 \\
\hline Clozapine & 33 & 44,100 & 1.27 & 45,100 & 1.23 & 42,400 & & 37,0 & 0.84 & 35,300 & 0.81 & 34,000 & 0.77 & 30,900 & 0.75 & 30,200 \\
\hline Queti & 1.04 & 37,800 & 1.05 & 39,400 & 1.05 & 41,300 & 1.11 & 43,700 & 1.24 & 42,800 & 1.31 & 41,300 & 1.37 & 37,600 & 1.47 & 36,500 \\
\hline Levomepromazine & 1.31 & 49,200 & 1.24 & 51,800 & 1.10 & 54,600 & 1.31 & 56,100 & 1.51 & 53,900 & 1.68 & 52,100 & 1.73 & 46,500 & 1.75 & 46,100 \\
\hline Lomatrigine & 1.05 & 22,400 & 1.10 & 19,600 & 1.11 & 18,400 & 0.88 & 17,6 & 0.81 & 16,400 & 0.78 & 15,900 & 0.73 & 14,700 & 0.71 & 13,800 \\
\hline nid & 1.24 & 24,800 & 1.17 & 23,300 & 1.06 & 23,100 & 1.02 & 22,500 & 0.95 & 21,100 & 0.90 & 20,800 & 0.84 & 18,900 & 0.80 & 18,300 \\
\hline & 1.14 & 15,900 & 1.19 & 18,800 & 1.21 & 22,800 & 0.97 & 27,400 & 0.92 & 26,900 & 0.87 & 26,400 & 0.82 & 24,800 & 0.79 & 19,200 \\
\hline & 1.34 & 13,700 & 1.33 & 12,700 & 1.30 & 11,700 & 1.34 & 8700 & 1.48 & 8100 & 1.56 & 7900 & 1.63 & 7700 & 1.60 & 7800 \\
\hline & 1.01 & 42,000 & 1.04 & 41,500 & 1.05 & 46,400 & 1.0 & 50,800 & 1.19 & 49,700 & 1.28 & 48,200 & 1.35 & 45,200 & 1.39 & 41,900 \\
\hline bamazepine & 0.95 & 38,400 & 0.98 & 40,900 & 1.02 & 26,400 & 0.94 & 45,300 & 0.87 & 46,100 & 0.83 & 45,600 & 0.80 & 40,100 & 0.78 & 37,900 \\
\hline Paroxetine & 1.18 & 40,500 & 1.14 & 34,700 & 1.09 & 46,400 & 1.14 & 48,800 & 1.27 & 48,300 & 1.35 & 47,700 & 1.47 & 43,500 & 1.53 & 40,300 \\
\hline Perfenazine & 1.23 & 53,500 & 1.20 & 55,100 & 1.15 & 59,300 & 1.18 & 65,100 & 1.27 & 63,200 & 1.42 & 61,200 & 1.49 & 58,300 & 1.51 & 51,100 \\
\hline Risperidon & 1.09 & 38,200 & 1.10 & 39,000 & 1.08 & 40,200 & 1.11 & 43,800 & 1.20 & 41,100 & 1.29 & 40,300 & 1.37 & 37,200 & 1.42 & 36,700 \\
\hline & 1.36 & 51,300 & 1.25 & 53,200 & 1.08 & 55,300 & 1.25 & 56,800 & 1.39 & 54,600 & 1.50 & 52,800 & 1.64 & 49,900 & 1.67 & 48,600 \\
\hline & 1.03 & 25,500 & 1.01 & 26,500 & 0.91 & 26,300 & 0.93 & 24,400 & 0.84 & 21,900 & 0.78 & 20,500 & 0.77 & 19,700 & 0.75 & 18,900 \\
\hline Zolpidem & 1.36 & 35,100 & 1.28 & 37,800 & 1.17 & 40,200 & 1.15 & 41,100 & 1.05 & 40,700 & 0.92 & 38,100 & 0.89 & 36,400 & 0.85 & 34,600 \\
\hline
\end{tabular}

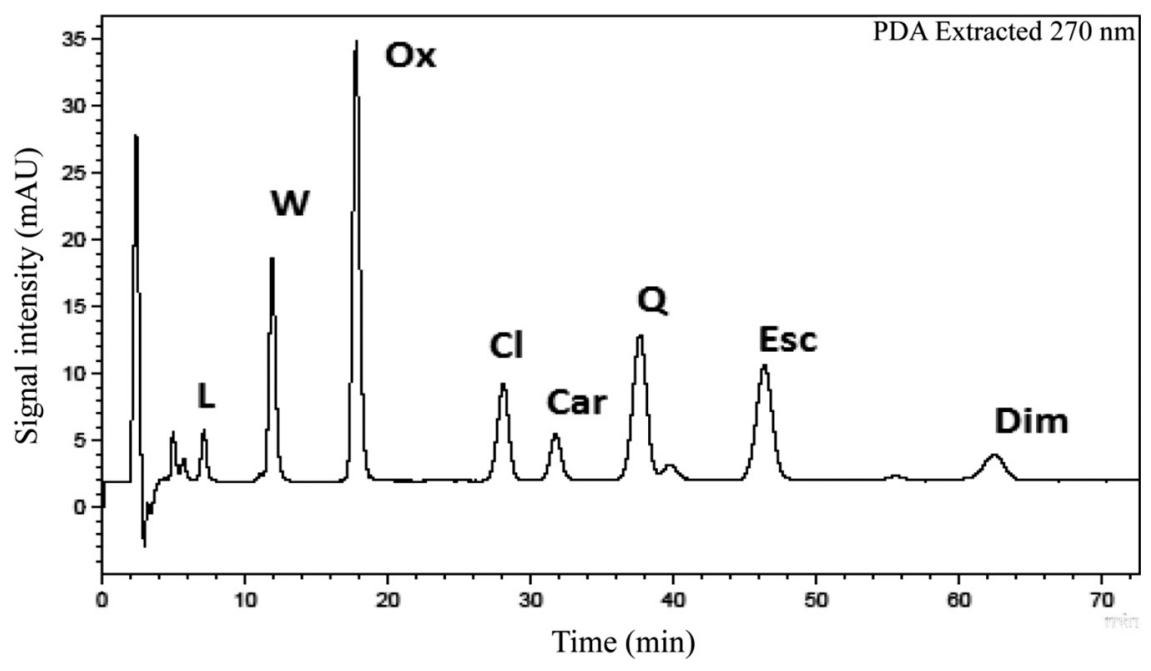

Figure 4. Chromatogram of psychotropic drug standards mixture obtained on the RP-Phe column eluted with a mixture of MeCN, water, and 0.5\% 1-butyl-3-methylimidazolium tetrafluoroborate 

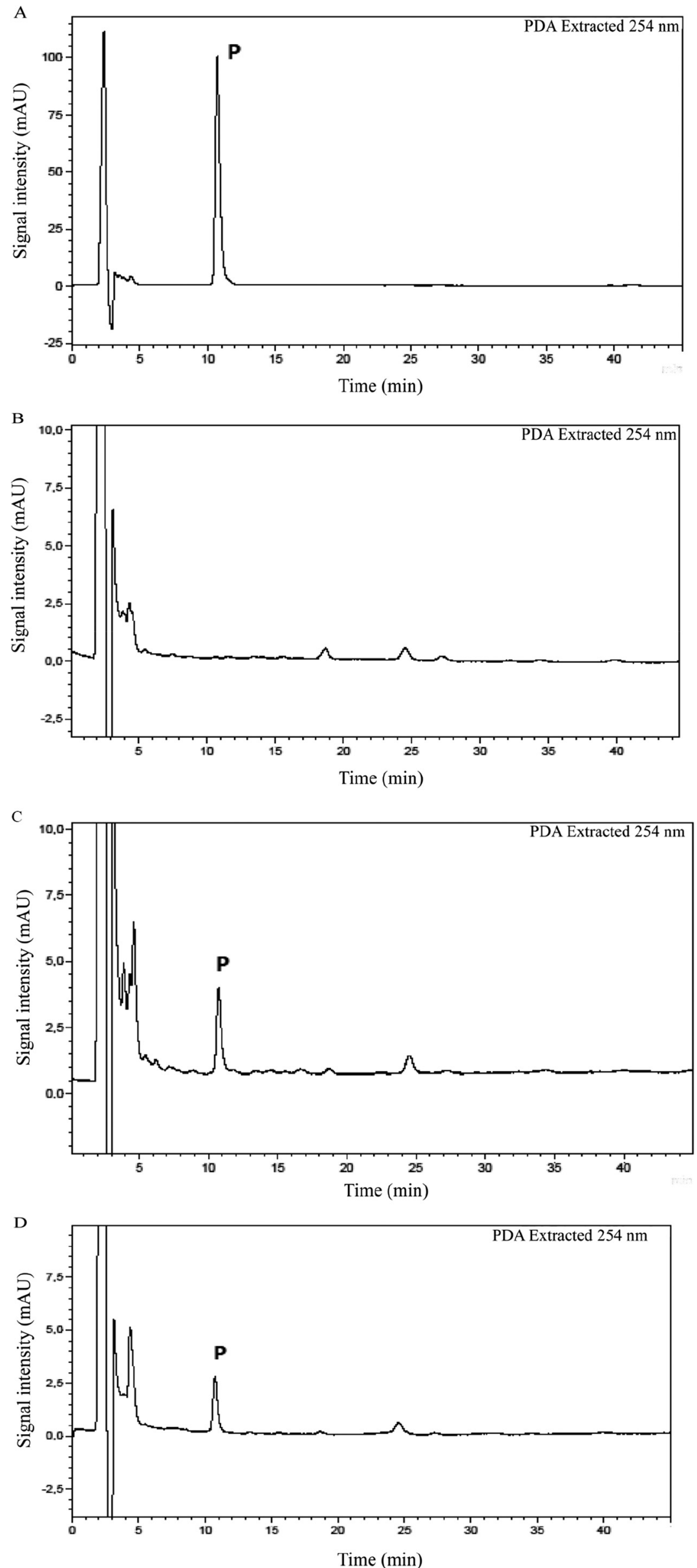

Figure 5. Chromatograms obtained on the RP-Phe column eluted with a mixture of MeCN, water, and 0.5\% 1-butyl-3-methylimidazolium tetrafluoroborate. A, Perazine standard; B, blank human serum; C, human serum fortified by $0.4 \mu \mathrm{g} / \mathrm{mL}$ of perazine; D, human serum from patient medicated by perazine 
these changes have not been such significant as these obtained when concentration of IL was changed. The most symmetrical peaks were obtained in mobile phase containing $25 \%$ or $30 \%$ of $\mathrm{MeCN}$; for 21 drugs, $A_{\mathrm{s}}$ values were optimal $\left(0.8<A_{\mathrm{S}}<1.5\right)$. Slight differences were also observed in system efficiency with the change of $\mathrm{MeCN}$ concentrations. For most cases, $N / m$ values decreased with the increase of $\mathrm{MeCN}$ concentration. For most of investigated compounds, the highest $\mathrm{N} / \mathrm{m}$ values were obtained with mobile phases containing $20 \% \mathrm{MeCN}$, but in the system, some psychotropic drugs were still strongly retained on RP-Phe column stationary phase $\left(t_{\mathrm{R}}>100 \mathrm{~min}\right)$. The sufficient system efficiency for most investigated drugs was obtained when concentrations of $\mathrm{MeCN}$ were in range $25-35 \%$. In mobile phases containing $25 \%$ or $30 \%$ of acetonitrile and in system containing $35 \% \mathrm{MeCN}$ for 17 and for 20 compounds, respectively, $\mathrm{N} / \mathrm{m}$ values were $>25,000$.

The influence of temperature on retention, peaks' shape, and systems efficiency was also investigated. The experiments were performed by using mobile phase containing $25 \% \mathrm{MeCN}$, water, and $0.25 \% \mathrm{IL}$ at temperatures in the range of $10-45{ }^{\circ} \mathrm{C}$. In applied system, linear decrease of retention with the increase of temperature has been obtained for all investigated psychotropic drugs according to van't Hoff's equation (Figure 3). These dependencies are typical for unionized compounds, which may indicate a predominant influence of ion-pairs formation on the retention in the chromatographic system. Separation selectivity was similar for most investigated drugs in all range of temperature.

Symmetry of peaks changed significantly with the change of temperature (Table 3). At temperatures 10 and $15^{\circ} \mathrm{C}$ (only peak for chlordiazepoxide was asymmetrical) for 13 and 16 compounds, respectively, excellent shape of peaks was obtained. When experiments were performed at 20 or $25^{\circ} \mathrm{C}$ for all investigated drugs, symmetrical peaks were observed, and for 18 and 17 compounds, respectively, symmetry of peaks was excellent. Further increase of temperature caused deterioration in the shape of the peaks especially when it was higher than $45^{\circ} \mathrm{C}$ when only for 6 drugs symmetrical peaks were obtained.

High systems efficiencies were obtained for most psychotropic drugs in whole range of examined temperatures. For most compounds, $\mathrm{N} / \mathrm{m}$ values slightly increased with increase of temperature and reached its maximum at $25{ }^{\circ} \mathrm{C}$ and then decreased again. For example, for aripiprazole, at $10{ }^{\circ} \mathrm{C}, \mathrm{N} / \mathrm{m}$ was 55,300 ; at $25{ }^{\circ} \mathrm{C}, 64,800$; and at $45^{\circ} \mathrm{C}, 54,200$.

Usefulness of chromatographic system containing IL for analysis of basic psychotropic drugs was confirmed by application of it for analysis of mixture of psychotropic drugs' standards and for analysis of perazine in human serum from patient. For this purpose, mobile phase containing $25 \% \mathrm{MeCN}$, water, and $0.25 \% \mathrm{IL}$ was applied. Experiments were performed at $22^{\circ} \mathrm{C}$. Figure 4 presents chromatogram obtained for separation of mixture of psychotropic drug standards. All components of the mixture were separated, and symmetrical peaks were obtained.

The same chromatographic system was applied for analysis of perazine. Figure 5A shows chromatogram obtained for standard of perazine. In Figures $5 \mathrm{~B}$ and $5 \mathrm{C}$, chromatograms obtained for blank human serum and for human serum fortified by $0.4 \mu \mathrm{g} / \mathrm{mL}$ of perazine were presented. Before HPLC analysis, blank human serum and fortified human serum samples were prepared by procedure described in Experimental section. There was no significant interference for the drug and components of matrix. Chromatogram obtained for serum sample from patient medicated with perazine was presented in Figure 5D.

\section{Conclusions}

The mobile phases containing addition of IL 1-butyl-3methylimidazolium tetrafluoroborate as silanol blocker were successfully applied for analysis of basic psychotropic drugs by HPLC on RP-Phe column.

In mobile phases without addition of IL, the obtained peaks were asymmetrical and system efficiencies were low. The application of mobile phases containing even low concentration of IL $(0.05 \%)$ significantly improves both shape of peaks and system efficiency. The most symmetrical peaks, highest $N / m$ values, and sufficient separation selectivity were obtained for drugs on Polar $\mathrm{RP}$ column with mobile phase containing $25 \% \mathrm{MeCN}$, water, and $0.25 \% \mathrm{IL}$. This is due to the masking of residual free silanol groups on the surface of the silica-based stationary phases and creation of ion-pair between cations of analytes and anions of IL.

Additionally, application of stationary phases with $\pi-\pi$ interactions, e.g., containing phenyl groups such as RP-Phe column, allows double protection against deleterious interaction between basic analytes and free silanol groups.

Systematic optimization of retention behavior of the investigated drugs enables a choice of the best chromatographic systems for separation of mixture of psychotropic drugs and analysis of one of them (perazine) in human serum samples.

\section{References}

1. Urinovská, R.; Brozmanová, H.; Sistík, P.; Silhánc, P.; Kacírová, I.; Lemr, K.; Grundmann, M. J. Chromatogr. B 2012, 907, 101.

2. Ansermot, N.; Brawand-Amey, M.; Kottelat, A.; Eap, C. B. J. Chromatogr. A 2013, 1292, 160

3. Kellner, M.; Porseryd, T.; Porsch-Hällström, I.; Hansen, S. H.; Olsén, K. H. Aquat. Toxicol. 2015, 158, 165 .

4. Qi, L.; Duan, L.-M.; Sun, X.-H.; Zhang, J.; Zhang, Z.-Q. Biomed. Chromatogr. 2015, 29, 1535 .

5. Choong, E.; Rudaz, S.; Kottelat, A.; Guillarme, D.; Veuthey, J.-L.; Eap, C. B. J. Pharm. Biomed. Anal. 2009, 50, 1000.

6. Xiong, C.; Ruan, J.; Cai, Y.; Tang, Y. J. Pharm. Biomed. Anal. 2009, 49, 572.

7. Petruczynik, A.; Wróblewski, K.; Szultka-Młynska, M.; Buszewski, B.; Karakuła-Juchnowicz, H.; Gajewski, J.; Morylowska-Topolska, J.; WaksmundzkaHajnos, M. J. Pharm. Biomed. Anal. 2016, 127, 68.

8. Calabuig-Hernández, S.; García-Alvarez-Coque, M. C.; Ruiz-Angel, M. J. J. Chromatogr. A 2016, 1465, 98.

9. Tang, Y.; Sun, A.; Liu, R.; Zhang, Y. Anal. Chim. Acta 2013, 767, 148.

10. Ding, X.; Tang, Y.; Sun, A.; Liu, R. J. Sep. Sci. 2015, 38, 374.

11. Ubeda-Torres, M. T.; Ortiz-Bolsico, C.; García-Alvarez-Coque, M. C.; Ruiz-Angel, M. J. J. Chromatogr. A 2015, 1380, 96.

12. Han, D.; Wang, Y.; Jin, Y.; Row, K. H. J. Chromatogr. Sci. 2011, 49, 63.

13. Horak, J.; Maier, N.M.; Lindner, W. J. Chromatogr. A 2004, 1045, 43-58, doi: 10.1016/j.chroma.2004.05.096.

14. Taylor, T. Getting the Most from Phenyl Stationary Phases for HPLC, LC GC Chromatographyonline http://www.chromatographyonline.com/print/320727? page $=$ ful (accessed Sep 13, 2016). 